J. Biosoc. Sci., (2016) 48, S56-S73 (C) Cambridge University Press, 2016. This is an Open Access article, distributed under the terms of the Creative Commons Attribution licence (http:// creativecommons.org/licenses/by/4.0/), which permits unrestricted re-use, distribution, and reproduction in any medium, provided the original work is properly cited.

doi:10.1017/S0021932016000067

\title{
COMMUNITY CO-DESIGNED SCHISTOSOMIASIS CONTROL INTERVENTIONS FOR SCHOOL-AGED CHILDREN IN ZANZIBAR
}

B. PERSON*1, S. KNOPP $\dagger \$$, S. M. ALIף, F. M. A'KADIR ${ }^{\mathrm{a}}$, A. N. KHAMIS ${ }^{\mathrm{a}}$, J. N. ALI' ${ }^{\mathrm{a}}$, J. H. LYMO ${ }^{\mathrm{a}}$, K. A. MOHAMMED ${ }^{\mathrm{a}}$ AND D. ROLLINSON†

* Schistosomiasis Consortium for Operational Research and Evaluation, University of Georgia, Athens, Georgia, USA, †Department of Life Sciences, Natural History Museum, London, UK, †Department of Epidemiology and Public Health, Swiss Tropical and Public Health Institute, Basel, Switzerland, §University of Basel, Basel, Switzerland, $\Phi$ Public Health Laboratory - Ivo de Carneri, Pemba, United Republic of Tanzania and ${ }^{\mathrm{a}}$ Helminth Control Laboratory Unguja, Ministry of Health, Unguja, United Republic of Tanzania

Summary. Top-down biomedical interventions to control schistosomiasis in subSaharan Africa have had limited success, primarily because they fail to engage with the social, political, economic and ecological contexts in which they are delivered. Despite the call to foster community engagement and to adapt interventions to local circumstances, programmes have rarely embraced such an approach. This article outlines a community co-designed process, based upon Human-Centered Design, to demonstrate how this approach works in practice. It is based on initial work undertaken by social science researchers, public health practitioners and community members from the Zanzibar Islands, Tanzania, between November 2011 and December 2013. During the process, 32 community members participated in a qualitative and quantitative datadriven workshop where they interpreted data on local infections from S. haematobium and co-designed interventions with the assistance of a facilitator trained in the social sciences. These interventions included the implementation of novel school-based education and training, the identification of relevant safe play activities and events at local schools, the installation of communitydesigned urinals for boys and girls and the installation of community-designed laundry-washing platforms to reduce exposure to cercariae-contaminated fresh water. It is suggested that the a community co-designed process, drawing from Human-Centered Design principles and techniques, enables the development of more sustainable and effective interventions for the control of schistosomiasis.

\footnotetext{
${ }^{1}$ Corresponding author. Email: bobbieperson@gmail.com
} 


\section{Introduction}

Urogenital schistosomiasis can contribute to anaemia, growth stunting, cognitive impairment, decreased work capacity and the potential for significant long-term health consequences such as bladder cancer and infertility (Barsoum, 2013; Knopp et al., 2013a). The disease disproportionately affects resource-poor people and contributes to lifelong disadvantages for those affected (King, 2010; Utzinger et al., 2011; Barsoum, 2013).

Interventions to combat schistosomiasis have typically been vertical, pathogenfocused efforts, with population-based chemotherapy programmes promoting the consumption of the drug praziquantel to reduce morbidity. These interventions are often implemented with limited understanding and attention to the socio-cultural, economic, religious, historical and political factors that shape local understandings and responses to endeavours to prevent and control schistosomiasis (Mwanga et al., 2004; Parker et al., 2008, 2012; Allotey et al., 2010; Mwanga \& Lwambo, 2013; Parker \& Allen, 2014). By encouraging local people to actively participate in the process of designing interventions, it is possible to draw upon socio-cultural norms and to develop locally tailored behaviour change messages, activities and structural interventions.

In the past 25 years, public health community-based participatory research has emerged as a research paradigm and an orientation to research that has focused on relationships between academic and community partners, with principles of mutual learning and long-term commitment that incorporated theories, participation and practices into the research efforts (Israel et al., 1998). Similarly, participatory methodologies that use stakeholder participation were also being explored in the area of industrial design to improve computer software and information technology to advance consumer usability, develop health care services and systems to create more user-focused models of care, advance service engineering to improve tracking of home-based nutritional status and, more recently, Human-Centered Design to enable co-designed social innovations for public health and development by community members, stakeholders and researchers (Schuler \& Namioka, 1993; Spinuzzi, 2005; Vink et al., 2008). While similar to traditional participatory research methods, the Human-Centered Design process also includes the participation of community members in the process of co-designing a specific product or intervention. In other words, it is grounded in design thinking that attempts to match the needs of users with easy-to-use products and systems (IDEO, 2011). Human-Centered Design relies upon a prescribed set of processes that can help researchers and community members to explore their basic needs together in new ways, create innovative products and solutions to meet these needs, and deliver the subsequent solutions with greater potential for sustainability (IDEO, 2011). The Human-Centered Design process starts with acquiring a thorough understanding of user needs through the use of several qualitative research methods for data gathering. The findings are then used to frame the problem. A number of techniques are subsequently used to build a deeper understanding on the part of the researcher, focusing on the social-cultural factors that might contribute to users finding solutions to their own problems. Concepts and ideas that address the identified problems are subsequently turned into prototype models and tested by the user. The early prototypes are unpolished and quickly put together so that the Human-Centered Design team can identify any specific challenges that need addressing and ensure that it most effectively solves the problem while best meeting the user's needs. 


\section{Schistosomiasis in Zanzibar}

Urogenital schistosomiasis, caused by infection with Schistosoma haematobium, is known locally in Zanzibar (Unguja and Pemba Islands) as kichocho. Disease prevalence in the past was estimated to be more than $60 \%$ of the entire population in Pemba and more than $50 \%$ in high-risk areas in Unguja (McCullough \& Kraft, 1976; Mgeni et al., 1990). Since then, the prevalence of $S$. haematobium infection has fallen to below $10 \%$. However, transmission hotspots remain (Stothard et al., 2009; Knopp et al., 2013a, b), and visible haematuria, microhaematuria and urinary tract pathology associated with urogenital schistosomiasis are still common (Savioli et al., 1990; Sousa-Figueiredo et al., 2008; Stothard et al., 2009). Successes in the reduction of infection prevalence and intensity have been mainly attributed to the regular administration of preventive chemotherapy on the islands, but a considerable improvement in socioeconomic living standards, including access to safe water and household latrines, also need to be considered (Stothard et al., 2009; Guidi et al., 2010; Knopp et al., 2013b). To eliminate urogenital schistosomiasis transmission on Unguja Island and schistosomiasis as a public health problem on Pemba Island, the Zanzibar Elimination of Schistosomiasis Transmission (ZEST) alliance was formed in 2011 (Knopp et al., 2012, 2013b). At the same time, a randomized intervention trial with three study arms, each including fifteen administrative areas (shehias) on both Unguja and Pemba, was implemented to compare the impact of preventive chemotherapy as administered by the Ministry of Health (Arm 1) with preventive chemotherapy as administered by the Ministry of Health plus snail control (Arm 2), and with preventive chemotherapy as administered by the Ministry of Health plus community-designed behaviour change interventions (Arm 3) (Knopp et al., 2012).

This article describes the use of Human-Centered Design in a community co-designed process for the control of schistosomiasis for the first time. It shows how local knowledge, experiences and creativity may be drawn upon to co-create practical, community-owned behavioural and structural interventions to interrupt the transmission of $S$. haematobium. Although a number of difficulties were encountered, it is suggested that the approach will enable more sustainable and effective interventions to be designed.

\section{Study setting}

The Zanzibar Archipelago is a semi-autonomous part of the United Republic of Tanzania and includes the two large islands of Unguja and Pemba with an estimated population of 1,264,327 people in 2012 (National Bureau of Statistics \& Office of Chief Government Statistician Zanzibar, 2013). Unguja and Pemba are divided into six and four districts respectively. Each district is subdivided into smaller administrative units, known as shehias, governed by a community leader (sheha). Zanzibar's main industries are spices, raffia and tourism. The majority of the population is Muslim. In 2012, upper respiratory tract infections, pneumonia, diarrhoea, skin diseases and ear, nose and throat diseases were reported by the Ministry of Health to be the top five most prevalent diseases in Zanzibar. Intestinal worm infections were ranked seventh (Ministry of Health et al., 2013). Interestingly, urogenital schistosomiasis, lymphatic filariasis and malaria were considered to be major public health problems until the beginning 
of the $21^{\text {st }}$ century, but the scaling-up of interventions in recent years along with broader socioeconomic changes has meant that these tropical diseases have been reduced to levels where elimination may be achievable (WHO, 2001; Mohammed et al., 2006; Smith et al., 2011; Knopp et al., 2012, 2013a). Infection hot-spots, however, do still occur (Knopp et al., 2013b; Hardy et al., 2015). In the case of urogenital schistosomiasis, the overall prevalence in Zanzibar was recently reported to be less than $10 \%$, with infection intensities also being found to be low in 2013, but in some shehias more than $20 \%$ of the surveyed population (children and adults) were infected with S. haematobium (Knopp et al., 2013a).

\section{Human-Centered Design}

Human-Centered Design is a co-design process based on the assumption that community members are experts who know best about workable solutions for their own problems - in this case preventing schistosomiasis (IDEO, 2011). The process is comprised of a set of tools, techniques, methods, tips and worksheets enabling participants to create and implement strategies towards the elimination of schistosomiasis. The Human-Centered Design process starts with the people for whom the behavioural intervention is intended to influence and comprises three major phases: hear, create and deliver. Drawing upon these underpinnings of Human-Centered Design we moved forward with our community co-design process.

\section{Phase 1: Hear}

Data collection. Phase 1 is the discovery process where qualitative interviews and discussions are conducted to hear what community members have to say. In this early stage of the process, research is both generative and evaluative. It is used to learn about people's current knowledge, attitudes, perceptions, practices and behaviours (IDEO, 2011); and it provides an opportunity to evaluate people's responses to potential new ideas and intervention strategies against schistosomiasis.

During Phase 1, a senior social scientist and skilled qualitative researcher trained seven bilingual Kiswahili- and English-speaking research assistants from the Ministry of Health in Unguja and the Public Health Laboratory in Ivo de Carneri, Pemba, to collect narratives from community members on the topic of schistosomiasis. Data collection tools, including topic guides and a written statement outlining the rationale for the project, along with informed consent documents, were translated into Kiswahili, pre-tested and modified to adapt to local linguistic and cultural idioms by the social science research team. Topic guides explored current perceptions, knowledge, attitudes, social norms and specific behaviours among children in Zanzibar that put them at risk for schistosomiasis. Additionally, community members' experiences, ideas and thoughts about how to change risky child behaviours were explored. Cultural factors, gender roles, influential communication channels and decision-making processes were also explored during the interview sessions.

Initially, the social science team conducted brief discovery sessions with influential religious and political leaders in the communities to explain the study, gain permission to 
undertake the work and develop respectful relationships. The team proceeded to gather narrative data from 35 school-based discussions with children, five focus group discussions with community figureheads and in-depth interviews with 25 teachers and sixteen parents. Structured observations of children at fresh water sites in and near villages were also conducted by the team. The exploratory qualitative research was conducted in five randomly selected shehias on Unguja (Chaani, Dole, Kilombero, Mwera and Uzini) and in two shehias on Pemba (Chambani and Kizimbani), among the fifteen selected study shehias on each island, in 2011. Perceptions and practices associated with transmitting, having, treating and preventing schistosomiasis were explored. The team collected children's and adults' stories, conducted observations and gathered opinions, insights and experiences to gain a deeper understanding of the needs, challenges and constraints to specifically prevent children from urinating in, and coming into contact with, contaminated natural open fresh water bodies. Data were collected until saturation was reached. That is, data collection ceased when no new data were emerging from the interviews or discussions.

Triangulation of data was obtained by using multiple groups, multiple methods and a literature search. Kiswahili audio recordings were translated into English transcripts and entered as Microsoft WordC documents into ATLAS.ti to facilitate text searching, data coding and analysis. Due to the paucity of qualitative research on schistosomiasis among Zanzibari school children, the team used a modified grounded theory (Strauss \& Corbin, 1998; Charmaz, 2003). Due to time constraints and on-going data collection tasks, Person (the first author and a senior social scientist) was the primary data coder, with verification of interpretive codes being undertaken by the research assistants. Open, axial and selective coding was used to analyse the children's group discussions, focus group discussions and in-depth interview narratives (Miles \& Huberman, 1994; Strauss $\&$ Corbin, 1998; Charmaz, 2003). A coding frame was developed through open coding, and a word-by-word analysis was used to identify, name and categorize explanations and descriptions of the day-to-day reality of participants as related to schistosomiasis. Axial coding - the process of relating codes to each other, via a combination of inductive and deductive thinking - was applied for analysis of specific emergent themes, across themes and for the relationships between themes (Miles \& Huberman, 1994; Strauss \& Corbin, 1998). Over the course of data collection, emergent themes became redundant, suggesting that all major themes had been identified. An analysis matrix served as a framework for analysing the data.

Findings. There were several major findings that emerged from the qualitative inquiry in the Phase 1 discovery process. First, it was widely stated in the in-depth interviews and discussion groups that girls were at little risk of disease. An older student reported, for example:

Girls don't void blood. They don't get kichocho.

Similarly, a parent said:

There are differences in the nature for men, kichocho symptom will appear quickly but it will take some time for them to appear in girl and for girls it is not common. They don't get disease...it is different than with boys. 
Teachers also reported that they themselves had received little to no education about the transmission, prevention, control or treatment of kichocho and they wanted to be trained to teach their students about the disease. A teacher from Pemba, said:

The first thing teachers need is a training so they can provide knowledge to the children about everything about kichocho.

Another teacher said:

We need to create a syllabus concerning health and kichocho. There also needs to be cooperation between teachers and doctor. Doctors need to follow-up at schools.

Narrative data established that children were at risk of infection because of their widespread domestic and recreational activities in natural open fresh water harbouring snail vectors capable of facilitating transmission. A parent reported:

There is only the river to play. There are not many places for older children to play. When they leave the house they may meet at the main road for chatting...the small ones when they come from school they go to Madrassa. But it is to the river where they get kichocho.

A teacher talking about risky domestic behaviours reported:

Small boys go there [to the river] with their parent. Others follow their friends in groups. Some children are told by their parents to go and bathe at the river because they should not spoil water at home. Water is very little and difficult to get...the boys will bathe for two hours or more in the river.

An older girl also said:

...girls go swimming, wash clothes and dishes [at the river] and take water home for domestic chores.

It was reported that children frequently urinate in fresh water sources. An older boy reported:

Boys don't like to urinate in the house. They urinate everywhere...even in the bushes. Boys aren't shy. They can urinate anywhere. Especially the young ones they will urinate in the water... an elder hides when finding a place to urinate.

A young girl told us:

You know girls are different from boys. They may be a little shy, but they do urinate in the water, but not like boys. Girls will hide but boys will pour it openly.

A community leader told us that girls also urinate in the river:

If girls are in the river they will urinate there. If not in the water they will urinate at the side but if the urine has the organisms they will enter in the water.

In short, almost everyone reported that they had heard about kichocho, with information having been acquired through informal (family/friends) networks. Generally, people knew few biomedical facts about kichocho, but they reported that it was commonplace among children and sometimes occurred among adults. However, kichocho was rarely acknowledged as an infection with potentially severe health consequences such as bladder cancer or infertility. 


\section{Phase 2: Create}

In Phase 2, the social science research team partnered with community participants to feed back findings and create the behavioural intervention. The senior social scientist served as the primary facilitator for the co-design process. The team shared data findings from Phase 1 with community members and they worked together to make sense of the data by identifying patterns, defining opportunities and creating solutions, including a final intervention for school-aged children in their shehia. During Phase 2, the research team conducted two co-design workshops for developing a behavioural intervention with attention to the needs, goals and priorities of the community. Thirty-two community members (including teachers, students, parents, physical education coaches, shehas, assistant shehas and religious and political leaders) participated in the workshops. The team presented their findings, which were characterized as design challenges rather than problems, in the following way: How can we create awareness that schistosomiasis is a disease of girls as well as boys? How can we create a teacher's package for teaching about kichocho in schools? How can we create alternative play activities for children to avoid contact with contaminated rivers, streams and ponds? How can we create an accessible urinal for children to urinate safely instead of contaminating water sources? How can we create a safe laundry area where children can wash clothes without standing in the river?

Interventions were co-designed by utilizing data together to generate new ideas, creating sketches or models of the intervention components, and eliciting feedback about the prototype sketches and models, focusing on their feasibility as real solutions. After reviewing and discussing the data, workshop participants decided that creating awareness that kichocho was a girl's as well as a boy's disease was an essential, crosscutting educational topic that should be incorporated across all intervention activities. To facilitate the co-design process, participants were divided into four workgroups, each of which was responsible for designing an intervention that addressed one of the remaining four design challenges. Brainstorming encouraged the generation of new ideas. Sketches and models of intervention ideas (known as prototypes) were generated by workshop members and shared with the entire group in order to get feedback and further develop the idea.

The first workgroup decided that given the lack of knowledge, educational materials and interactive teaching tools, it was critical to develop training materials for government primary school and Quran school (Madrassa) religious teachers to provide for use in the classroom. The workgroup contributed to the participatory process by synthesizing and sharing their knowledge of the limited current teacher resources, brainstorming new classroom activities and proposing the development of new teaching approaches. They decided that teachers needed a Kiswahili educational flipchart on kichocho, interactive teaching demonstrations, new factual materials and tools and interactive role-plays for students. It was also decided that one to two teachers from each school needed annual training, with specialized educational activities to equip them to return to their schools and train all the other teachers in order to engage as many children as possible in prevention and control activities. The group reported the need for more effective teaching tools, rather than the one-way didactic information, education and communication materials typical of other public health programmes. 
The second workgroup worked on creating safe games, activities and educational play for children to avoid playing in natural fresh water where they might become infected with kichocho. They reviewed the data and shared stories on the current play practices of school-aged children during and after school; and they synthesized and discussed current resources in the schools and options for during- and after-school activities. In addition, they identified local games and developed new games for children. They also identified a variety of channels for interacting with children in and out of school. The workgroup created an outline for a special school event for children where alternatives to risky water-based play would be highlighted as safe games and activities, which they named 'Kichocho Safe Play Day'. Participants also created a set of 24 activity cards that described the necessary supplies and instructions for each activity. Each activity or game had a set of schistosomiasis educational questions to stimulate discussion about the transmission, prevention and treatment of kichocho.

The third workgroup worked on designing an accessible urinal for children to use instead of urinating in local natural fresh water sources. Participants reviewed diverse designs from the literature, explored images of a variety of urinals including the P-Tree - a temporary tree-friendly urinal that can be fixed on every tree using straps and lashings - to stimulate new ideas. Ultimately, the workgroup created table models of potential urinals out of cardboard, Styrofoam and other local materials. Phase 1 findings revealed that girls as well as boys urinated in fresh water sources when conducting domestic chores. The workgroup inspired designs for community male and female urinals made from bricks and cement, as well as an eco-friendly coconut urinal that could be made from local resources and was of a smaller size that could be permanently attached to trees or other structures for use by young boys.

The fourth workgroup worked on designing a safe laundry area where children can wash clothes without standing in the river. It was reported in Phase 1 that boys and girls washed their clothes in the river due to non-working taps and the fact that river water produces more suds for cleaning clothes. Structured observations noted that laundry activities were conducted in areas segregated by gender. It was also observed that young boys typically stood in the water for an extended length of time playing during the clothes-washing process, while young girls tended to sit along the bank, often in the water itself. Participants created washing platforms and slab prototypes out of cardboard, paper and other materials.

At the end of the day, each workgroup presented opportunities for intervening, and tangible solutions and potential table-size models for their specific design challenge. Participants, as a group, continued to brainstorm and refine ideas for new behavioural and structural solutions towards each design challenge until consensus was achieved on the major intervention components.

\section{Phase 3: Deliver}

In Phase 3, the research team worked with teachers and other participants to deliver the interventions designed during Phase 2 of the community co-design process workshop. The interventions designed in Phase 2 were chosen because they were desirable, and focused on what people needed and wanted. They also appeared to be feasible within the local context. Finally, the interventions were practical for the study 
and financially sustainable following the cessation of the project. The workgroup in Phase 2 had identified the major intervention components and in Phase 3, beginning in October 2012, the social science research team worked with community members to deliver the interventions that were identified: classroom-based education; kichocho safe play day events; male and female urinals; and washing platforms. The on-going intervention is to be concluded in 2016 .

Classroom-based education. The research teams began with the school-based intervention component by training 60 schoolteachers and 30 religious teachers, known as Madrassa teachers, in a one-day teacher training session. The aim of the workshop in Year 1 was to increase knowledge about kichocho and provide new teaching methods and teaching tools to enable them to increase the likelihood of positive prevention behaviours among school-aged children. The training activities were focused on enabling teachers to share that knowledge with other teachers and students in their school. A Kiswahili kichocho flipchart (which contained photographs of local transmission sites, snail vectors, children engaging in risk activities, diagnostic lab techniques and treatment options) was designed, pre-tested and produced (see Fig. 1). It was finally provided to teachers at each of the 30 government schools and 30 Madrassa schools in the fifteen study shehias on each island. Teachers were also trained in new interactive teaching methods on kichocho, role-plays and safe play activities and games. They were asked to: use new teaching approaches with other teachers and in their classroom, to teach all students in grades one to six, to discuss kichocho once a week with all their students and to involve members of one school club in kichocho education activities to serve as peer educators and role models.

The teachers initially trained by the research teams in November 2012 returned to their schools and presented the teacher training as a locally designed training developed by teachers like themselves with input from students, parents and other people from the community. School-based process data were collected in 2012 and 2013. At the time of data collection, the trained teachers had trained 678 from a total of 761 registered teachers

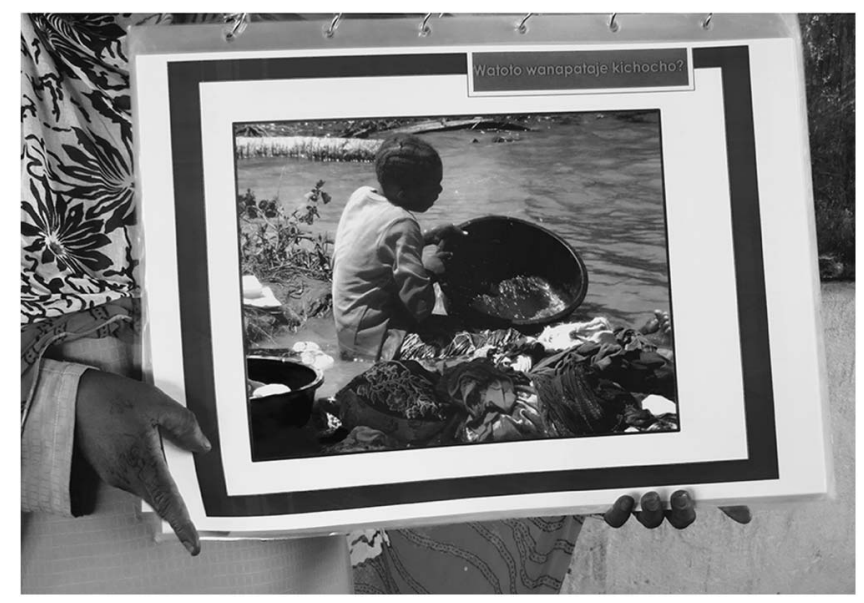

Fig. 1. Interactive educational schistosomiasis flipchart. 
in the study shehias. Teachers who did not receive the training were either new or absent on the day of the school-based training. The 678 teachers trained during the school-based trainings conducted at least one, but typically more than one, kichocho educational session with 27,819 children from a total of 30,034 possible students registered in the Unguja and Pemba study schools. Students who were absent on the days of the educational sessions or new to the school at the time of data collection might have missed these sessions. The research team, with help from the originally trained teachers, also trained students in school-based environmental and health clubs at each school.

Kichocho Safe Play Day events. Workshop participants decided in Phase 2 that Kichocho Safe Play Day events should be conducted twice a year at each study school. The aim of these events was to highlight and educate safe play alternatives to risky, contaminated water play. The research teams worked with the trained teachers to create a fun-filled behaviour-change day that allowed students to participate in safe play games and activities. As shown in Fig. 2, the tug-of-war game was identified as a safe play activity. Children who fell on the ground were considered as having fallen into the river and now had kichocho. They then had to answer five kichocho questions: What is in the river that causes kichocho? What are the symptoms of kichocho? Where do you go to get treatment for kichocho? What is the medicine that treats kichocho? How do you prevent getting kichocho in the future?

Fifty-two Kichocho Safe Play Day events were conducted in each of the fifteen study schools on both islands, with a total enrolment of 30,575 students during the first year of intervention activities. Teachers chose games and activities from the 24 activity cards designed during the co-design workshop and also created new games, role-plays, skits, songs and poems about kichocho that were presented to the entire school. Parents were also encouraged to attend in order to learn about the severe health consequences of this chronic disease and how to prevent this in their children.

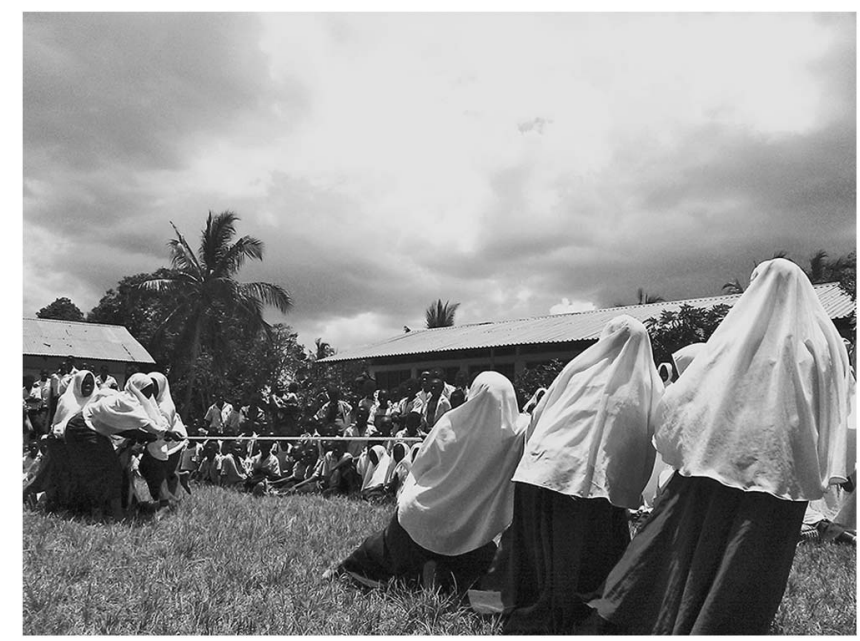

Fig. 2. Tug-of-War safe play educational activity. 
Male and female urinals. Passing S. haematobium eggs in urine into fresh water bodies where the intermediate host snails thrive is part of the parasite's transmission life cycle. The installation of urinals close to natural open fresh water bodies might help interrupt transmission. A local mason, who participated in the workshop, created and worked with the research teams to install one female and one male urinal in one village in each of 29 study shehias, as shown in Fig. 3. Since two shehias in Unguja shared the same natural fresh water body, only one pair of urinals was installed for both of them. A prototype male and female urinal was constructed in one shehia near a school where school children and local community members could provide feedback into the design, use and maintenance of the community urinals. The urinals were modified to adhere to Muslim culture practices. A local artist was hired to paint the structures, identifying them as male and female urinals, along with inscribing positive prevention practices and health-seeking messages for children who urinate blood. The urinals were installed near a known transmission hotspot where the likelihood of disease transmission was high. The research teams provided supplies for installing cement urinals and each shehia provided community member labour to build the urinals under the guidance of the mason who participated in the co-design workshop. A local carpenter also presented the teams with a prototype small, eco-friendly coconut urinal for young boys.

An education session was conducted with local village members with an emphasis on the importance of having children and adults urinate in the urinals instead of urinating in fresh water. The transmission of kichocho was explained and the actual snails that serve as intermediate host of the parasite were shown to the community members.

As part of the monitoring and evaluation of the intervention study, the research team members monitored and regularly collected data on community use, vandalism attempts and cleanliness of the male and female urinals. During the first six months following installation, for example, rubber tubing coming out from the urinals was stolen from several of the urinals. To prevent theft, they were subsequently covered with cement. Wooden hand-painted kichocho signs designed and produced by local artists, and nailed

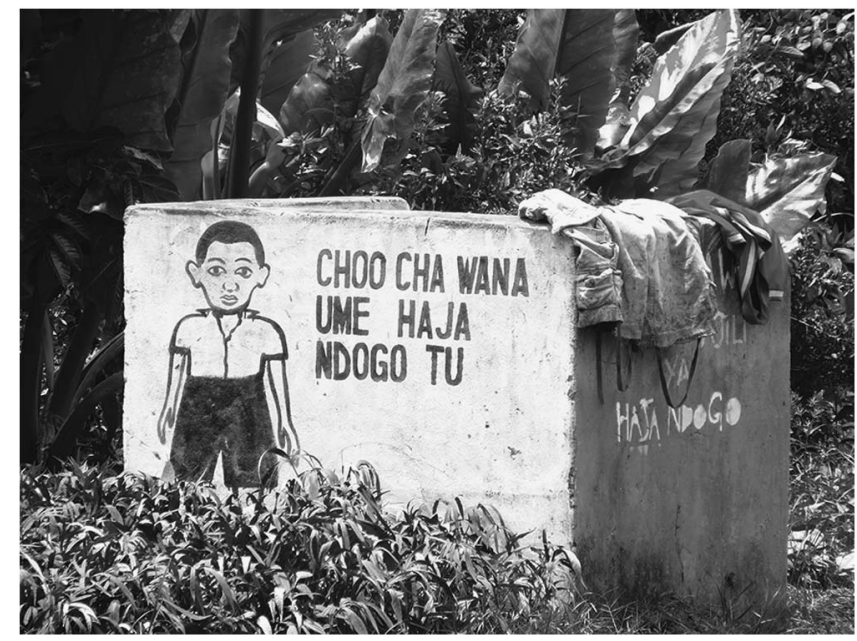

Fig. 3. Community-located male urinal. 
to trees at transmission hotspots, helped remind people of the risks of entering kichocho-contaminated water. Despite being placed high up in trees to prevent theft, these signs were often taken down and the wood used for stoves and other household needs. The signs were expensive and a safe way of securing them was not identified so community members decided not to replace them when stolen.

Washing platforms. Washing clothes in contaminated fresh water sources is a major risk behaviour of children and adults. In Zanzibar, young boys and girls wash their own clothes. While girls were noted to sit at the edge of the water or take a bucket of water further away from the riverbank, boys tended to stand in the infected rivers for longer periods of time playing, fishing and bathing while washing their clothes. Several different washing platforms and washing slabs were thus suggested and designed to accommodate a variety of environmental situations. The finally selected washing platform type was designed as a low circular design slab with a drainage trough, where women and children can sit, scrub laundry and socialize. Men and women share the platforms by washing laundry in a segregated fashion at different times of the day. These washing platforms are currently being installed in villages in each shehia where clean tap water or well water for public access exists, as shown in Fig. 4.

\section{Critical reflections and challenges ahead}

The behaviour-change component applied in the ZEST randomized intervention trial aims to interrupt schistosomiasis transmission by changing risk behaviours of children in Zanzibar. Through the Human-Centered Design process, it was possible to leverage robust local knowledge, experiences and creativity to create usable, complimentary and community-owned behavioural and structural interventions that can help to interrupt schistosomiasis transmission in the Muslim study communities in Zanzibar.

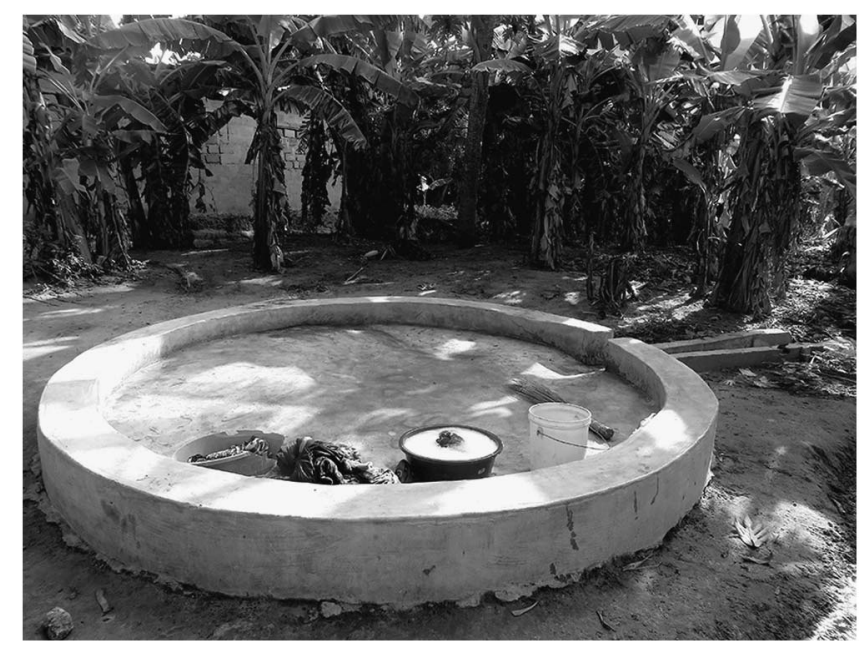

Fig. 4. Newly installed washing platform. 
The following challenges were faced and lessons were learned, which might be of consideration and help for establishing behavioural interventions in other parts of the world where sustainable control or elimination of urogenital schistosomiasis is the goal.

\section{Human-Centered Design qualitative research process}

The Human-Centered Design process of co-designing community interventions described in this article was a novel approach for the two research teams in Unguja and Pemba, as well as the community members. The qualitative formative research process required a professional social scientist to train the inexperienced teams in qualitative methods, act as the facilitator of the Human-Centered Design process and serve as a role model for interactions between the teams and community members. A written qualitative methods protocol and on-going guidance from the senior social scientist provided support for improving skills of the team members. Staff proficiency in qualitative methods increased over time resulting in new and informative knowledge from members of the study communities.

The social science research team engaged children through discussions and drawing pictures. Many children were shy at the beginning of group discussions, had never used crayons to draw pictures, and had a desire to please adults. This was a challenge for the team members because there is typically a cultural expectation that a child does as they are told. It was a new experience to encourage children to be creative and to share their ideas and thoughts. Each team member worked with a small group of children, guiding them through an initial free drawing session, which helped to lessen this problem. It allowed the shyer children to draw, and to become more comfortable with sharing their ideas prior to being asked to draw a picture about kichocho. Some adults initially offered short or uninformative answers. In common with many of the children, there was a desire to provide socially acceptable answers. The biggest challenge facing the team was thus to create the space to be able to recognize an unexplored, but potentially new and useful concept. This was resolved by reviewing and debriefing the interview sessions and practising how to probe further, thus identifying new areas that needed to be explored in future interviews. As the team became more comfortable with the interview process, members of the community provided more detailed, robust narrative data.

\section{Human-Centered Design intervention process}

In the co-design process it is posited that 'people intended to use a future technique, product or situation ... should play a substantial role in designing it' (Schuler \& Namioka, 1993). In this study, during the whole designing process, the professional social scientist served as a facilitator interfacing between the social science research team and the community as the user and designer. A great deal of skill was required by the facilitator to guide the co-design process and stimulate creative solutions. It was necessary to train the team to set aside their typical role as authority figures and to become more empathetic to the experiences, perceptions and practices of the children and adults they were trying to assist. The interactive discussion techniques and creative processes were new for both the team and participants in the workshop. The facilitator served as a role model for team members in the first community workshop for 
facilitating participatory activities, which the team was able to emulate during the second workshop.

Community members were not always knowledgeable of the disease process. Some were not able to express their needs or had no ideas about how to address their needs, and some were not willing to talk about their needs or present new ideas or interventions. There was an initial tendency to voice the potential problems of all new ideas rather than focus on the task of finding new solutions to local problems. By showing appreciation to those who voiced new ideas, openly discussing the potential benefits of a new idea and discussing the importance of local ownership, people were gradually encouraged to explore new possibilities.

Few people were comfortable with the initial creative design process, with many requiring a great deal of coaxing and positive reinforcement for their participation. Working through visual drawings and storytelling reduced the inhibition of many children and adults to participate in activities, exercises and processes. Standing up, rather than sitting down, during the interactive design exercises encouraged greater participation. Participants used paper, coloured pens, tape, sticky notes, drawing and model-building activities. Similar to other community-based participatory projects, the social science research team observed how participants became increasingly engaged with the process of creating interventions that were adapted to their own particular cultural practices and needs. While initially reluctant to participate, they became excited and enthusiastically engaged in the co-design processes and further supported the implementation of the intervention components in their communities.

The community co-design process allowed schoolteachers and Madrassa teachers to influence the classroom-based schistosomiasis educational curriculum for school-aged children. Teachers were very receptive to co-designing teaching activities, interactive teaching methods, new information materials, demonstrations and classroom evaluation techniques. Madrassa religious teachers were especially receptive to the process because this was the first time they had been asked to be involved in a health intervention study where their opinions and ideas were valued. They also made valuable suggestions about how to reach school-aged children who do not attend government schools.

Children in Zanzibar have few options for play activities other than the river. During the community co-design process, students provided critical insights and suggestions associated with acceptable safe play options. An open dialogue between students and teachers resulted in the development of a Kichocho Safe Play Day in each school. Traditional games and activities, favourites of children, were augmented with new kichocho educational games and activities. The students in the process expressed ownership of the Kichocho Safe Play Day because of their contributions to the planning process and a great enthusiasm for the new kichocho games and activities. They expressed optimism that alternative play options could reduce children's contact with contaminated water.

Urinals and washing platforms were planned as structural interventions to support the reduction of risky behaviours and the promotion of positive behavioural change. These structural interventions - the newest concepts for the facilitator, team and workshop participants - required the most discussion. The facilitator had to consider the local customs along with ritual washing and urination practices. For example, in Zanzibar, women and men wash their own clothes in segregated settings. Consideration was given to children, the primary audience of the intervention, tempered by the 
expressed needs of adults in the design and placement of the community urinals. Small mosques are often built near open fresh water sources, which are potential transmission sites for schistosomiasis. The requirement of ritual washing prior to prayers, often conducted in the respective streams and ponds, might contribute to the acquisition of infection. Placing urinals (which have private walled structures) near the small mosques allowed children and adults to urinate and wash in private prior to prayers, which was greatly appreciated. It was also determined that washing platforms would be best placed in areas with public access to clean water, i.e. taps, wells or boreholes, to encourage safe laundry practices. Washing laundry was often reported as a time for socialization and thus a platform that allowed for comfortable sitting was desirable. Powdered soap and bar soap were both widely used and the desire for a sturdy area upon which to scrub clothes was articulated.

Overall, the Human-Centered Design, community co-designed process required a great deal of support from the senior social scientist due to the inexperience of the local staff in social science methods, new community engagement approaches and the requirement of communication skills that differed from the cultural norm. A team of more experienced individuals may not need the level of support and role modelling described in this article. That said, members of the team enthusiastically embraced their skills, roles and relationships with participants and they quickly saw great value in the new process as compared with previous top-down, biomedical approaches.

\section{Conclusion}

Well-intentioned schistosomiasis prevention and control programmes, relying on vertical, biomedical interventions and the voluntary co-operation of villagers, have been fraught with difficulties. They often result in less than desirable drug uptake (Allen \& Parker, 2011; Parker \& Allen, 2011; Muhumuza et al., 2013; Tuhebwe et al., 2015). This is partly due to the implementation of protocols that have been developed without consideration of local circumstances or inputs from the very people who the interventions are supposed to be helping. Public health researchers and programme staff have a responsibility to move beyond paternalistic approaches and involve a wide range of local people in the development of schistosomiasis prevention and control interventions, not least because they are so knowledgeable of the local situation and are best placed to know what kind of intervention will - and will not - work. The research presented in this article describes a community engagement process that enables local people to come together and contribute to a schistosomiasis prevention and control effort. It shows how different kinds of people can work together through facilitated dialogue to generate new complimentary ideas for community-owned, behavioural and structural interventions tailored to their own social, religious and cultural norms. The process also established the credibility of the social science research team as trusted partners who listened to, and valued, the input of community members. Although the Human-Centered Design process shows great promise in developing and implementing community co-designed interventions, the success of the interventions in reducing schistosomiasis transmission will ultimately be determined in the coming years of the ZEST study. 


\section{Acknowledgments}

The authors are grateful to the school children, their teachers and community members of the study communities for their co-operation and commitment to eliminating kichocho in Zanzibar. They would also like to express their thanks to Sam van Veluw for providing a P-tree urinal that served as an excellent example to the local carpenters. This study received financial support from the University of Georgia Research Foundation, through the Schistosomiasis Consortium for Operational Research and Evaluation (SCORE), which is funded by the Bill and Melinda Gates Foundation (Sub-award No. RR374-053/4893206). SK is financially supported by sub-award No. RR374-053/ 4893196.

\section{References}

Allen, T. \& Parker, M. (2011) The 'other diseases' of the Millennium Development Goals: rhetoric and reality of free drug distribution to cure the poor's parasites. Third World Quarterly 32(1), 91-117.

Allotey, P., Reidpath, D. D. \& Pokhrel, S. (2010) Social sciences research in neglected tropical diseases: the ongoing neglect in the neglected tropical diseases. Health Research Policy and Systems 8(1), 32.

Barsoum, R. S. (2013) Urinary schistosomiasis: review. Journal of Advanced Research 4(5), 453-459.

Charmaz, K. C. (2003) Grounded theory: objectivist and constructivist methods. In Denzin, N. K. \& Lincoln, Y. S. (eds) Strategies of Qualitative Inquiry. Sage Publications Ltd, Thousand Oaks, CA, USA, pp. 249-291.

Guidi, A., Andolina, C., Ame, S. M., Albonico, M., Cioli, D. \& Haji, J. H. (2010) Praziquantel efficacy and long-term appraisal of schistosomiasis control in Pemba Island. Tropical Medicine \& International Health 15(5), 614-618.

Hardy, A., Mageni, Z., Dongus, S., Killeen, G., Macklin, M. G., Majambare, S., Ali, A. et al. (2015) Mapping hotspots of malaria transmission from pre-existing hydrology, geology and geomorphology data in the pre-elimination context of Zanzibar, United Republic of Tanzania. Parasites \& Vectors $8(1), 1-15$.

IDEO (2011) IDEO Human Centered Design Toolkit, $2^{\text {nd }}$ Edition. IDEO.

Israel, B. A., Schulz, A. J., Parker, E. A. \& Becker, A. B. (1998) Review of community-based research: assessing partnership approaches to improve public health. Annual Review of Public Health 19(1), 173-202.

King, C. H. (2010) Parasites and poverty: the case of schistosomiasis. Acta Tropica 113(2), 95-104.

Knopp, S., Becker, S., Ingram, K., Keiser, J. \& Utzinger, J. (2013a) Diagnosis and treatment of schistosomiasis in children in the era of intensified control. Expert Review of Anti-Infective Therapy 11(11), 1237-1258.

Knopp, S., Mohammed, K. A., Ali, S. M., Khamis, I. S., Ame, S. M., Albonico, M. et al. (2012) Study and implementation of urogenital schistosomiasis elimination in Zanzibar (Unguja and Pemba Islands) using an integrated multidisciplinary approach. BMC Public Health 12(1), 930.

Knopp, S., Person, B., Ame, S., Mohammed, K. A., Ali, S. M., Khamis, I. S. et al. (2013b) Elimination of schistosomiasis transmission in Zanzibar: baseline findings before the onset of a randomized intervention trial. PLoS Neglected Tropical Diseases 7(10), e2474.

McCullough, F. S. \& Krafft, J. G. (1976) Schistosomiasis in Zanzibar and Pemba, Tanzania. Report on a mission 1 April-7 June 1975. In AFR/SCHIST/34. World Health Organisation, Geneva. 
Mgeni, A. F., Kisumku, U., Mccullough, F., Dixon, H., Yoon, S. \& Mott, K. (1990) Metrifonate in the control of urinary schistosomiasis in Zanzibar. Bulletin of the World Health Organization 68(6), 721-730.

Miles, M. B. \& Huberman, A. M. (1994) Early steps in analysis. Chapter in Qualitative Data Analysis: An Expanded Sourcebook. Sage Publications Ltd, Thousand Oaks, CA, USA, pp. $50-89$.

Ministry of Health, Planning Unit \& Health Management Information System (2013) HMIS. Zanzibar Health Bulletin - 2012. Ministry of Health, Zanzibar.

Mohammed, K. A., Molyneux, D. H., Albonico, M. \& Rio, F. (2006) Progress towards eliminating lymphatic filariasis in Zanzibar: a model programme. Trends in Parasitology 22(7), 340-344.

Muhumuza, S., Olsen, A., Katahoire, A. \& Nuwaha, F. (2013) Uptake of preventive treatment for intestinal schistosomiasis among school children in Jinja district, Uganda: a cross sectional study. PLoS One 8(5), e63438.

Mwanga, J., Magnussen, P. \& Aagaard-Hansen, J. (2004) Schistosomiasis-related perceptions, attitudes and treatment-seeking practices in Magu District, Tanzania: public health implications. Journal of Biosocial Science 36(1), 63-81.

Mwanga, J. R. \& Lwambo, N. J. (2013) Pre-and post-intervention perceptions and water contact behaviour related to schistosomiasis in north-western Tanzania. Acta Tropica 128(2), 391-398.

National Bureau of Statistics \& Office of Chief Government Statistician Zanzibar. (2013) 2012 Population and Housing Census: Population Distribution by Administrative Units; Key Findings. Dar es Salaam, Tanzania, p. 21.

Parker, M. \& Allen, T. (2011) Does mass drug administration for the integrated treatment of neglected tropical diseases really work? Assessing evidence for the control of schistosomiasis and soil-transmitted helminths in Uganda. Health Research Policy and Systems 9, 3.

Parker, M. \& Allen, T. (2014) De-politicising parasites: reflections on attempts to control the control of neglected tropical diseases in Uganda and Tanzania. Medical Anthropology 33(3), 223-239.

Parker, M., Allen, T. \& Hasting, J. (2008) Resisting control of neglected tropical diseases: dilemmas in the mass treatment of schistosomiasis and soil-transmitted helminths in north-west Uganda. Journal of Biosocial Science 40, 161-181.

Parker, M., Allen, T., Pearson, G., Peach, N., Flynn, R. \& Rees, N. (2012) Border parasites schistosomiasis control among Uganda's fisherfolk. Journal of Eastern African Studies 6(1), 97-122.

Savioli, L., Hatz, C., Dixon, H., Kisumku, U. M. \& Mott, K. E. (1990) Control of morbidity due to Schistosoma haematobium on Pemba Island: egg excretion and hematuria as indicators of infection. American Journal of Tropical Medicine and Hygiene 43(3), 289-295.

Schuler, D. \& Namioka, A. (1993) Participatory Design: Principles and Practices. L. Erlbaum Associates Inc., Hillsdale, NJ, USA.

Smith, D. L., Cohen, J. M., Moonen, B., Tatem, A. J., Sabot, O. J., Ali, A. \& Mugheiry, S. M. (2011) Solving the sisyphean problem of malaria in Zanzibar. Science 332(6036), 1384-1385.

Sousa-Figueiredo, J. C., Basáñez, M. G., Mgeni, A. F., Khamis, I. S., Rollinson, D. \& Stothard, J. R. (2008) A parasitological survey, in rural Zanzibar, of pre-school children and their mothers for urinary schistosomiasis, soil-transmitted helminthiases and malaria, with observations on the prevalence of anaemia. Annals of Tropical Medicine \& Parasitology 102(8), 679-692.

Spinuzzi, C. (2005) The Methodology of Participatory Design. Technical Communication 52(2), $163-174$.

Stothard, J., French, M., Khamis, I. S., Basáñez, M. \& Rollinson, D. (2009) The epidemiology and control of urinary schistosomiasis and soil-transmitted helminthiasis in schoolchildren on Unguja Island, Zanzibar. Transactions of the Royal Society of Tropical Medicine and Hygiene 103(10), 1031-1044. 
Strauss, A. \& Corbin, J. (1998) Basics of Qualitative Research: Techniques and Procedures for Developing Grounded Theory. Sage Publications Ltd, Thousand Oaks, CA, USA, pp. 1-217.

Tuhebwe, D., Bagonza, J., Kiracho, E. E., Yeka, A., Elliott, A. M. \& Nuwaha, F. (2015) Uptake of mass drug administration programme for schistosomiasis control in Koome Islands, Central Uganda. PLoS One 10(4), e0123673.

Utzinger, J., N'goran, E. K., Caffrey, C. R. \& Keiser, J. (2011) From innovation to application: social-ecological context, diagnostics, drugs and integrated control of schistosomiasis. Acta Tropica 120 (Supplement 1), S121-S137.

Vink, P., Imada, A. S. \& Zink, K. J. (2008) Defining stakeholder involvement in participatory design processes. Applied Ergononomics 39(4), 519-526.

WHO (2001) Filariasis elimination, Zanzibar. The Weekly Epidemiological Record 76, 401-408. 